\title{
Management of the Third Stage of Labor: How Delayed Umbilical Cord Clamping Can Affect Neonatal Outcome
}

\author{
Mitchell ]. Kresch, MD, FAAP ${ }^{1}$ \\ ${ }^{1}$ Division of Newborn Medicine, Department of Pediatrics, Penn State \\ Health Children's Hospital, Hershey, Pennsylvania \\ Am J Perinatol 2017;34:1375-1381.
}

\author{
Address for correspondence Mitchell J. Kresch, MD, FAAP, Division of \\ Newborn Medicine, Penn State Health Children's Hospital, 500 \\ University Drive, Mail Code H085, Hershey, PA 17033-0850 \\ (e-mail: mkresch@pennstatehealth.psu.edu).
}

\begin{abstract}
Keywords

- neonatal resuscitation

- umbilical cord

- transitional physiology

This review presents the effects of delayed umbilical cord clamping on neonatal transitional physiology. The effects of delayed cord clamping on short- and long-term neonatal outcomes are then discussed. There is ample evidence over the last 50 years that delayed cord clamping in preterm infants is beneficial for both short-term and long-term outcomes. Providing ventilation in the initial steps of neonatal resuscitation prior to clamping of the umbilical cord has a physiologic basis and results in better outcomes for newborns. The challenge now is to design equipment and strategies that can allow initial resuscitation very close to the mother while the umbilical cord is still attached to the placenta.
\end{abstract}

Management of the third stage of labor includes clamping of the umbilical cord after delivery of the infant, use of uterotonic medications (e.g., Pitocin) and controlled traction applied to the umbilical cord, and possibly uterine massage. This review will focus on the first step in the process, that is, cord clamping.

It is often useful to be familiar with the historical practice of medicine as it can influence decisions and evaluation of our current practice. Dr. A.C. Beck wrote in 1941, "Often the cord is clamped at once...this practice is reprehensible since it deprives the immature infant of considerable blood, which it otherwise might take up from the placental circulation... Blood in the umbilical cord should be stripped toward the fetus before the umbilical cord is ligated." ${ }^{1}$ The practice of immediate cord clamping after delivery of the newborn infant has recently been challenged by the American College of Obstetrics and Gynecology. The Committee on obstetric practice stated that the evidence now supports the routine delay in cord clamping for at least 30 to 60 seconds for all vigorous term and preterm neonates. ${ }^{2}$ In addition, the seventh edition of the Textbook on Neonatal Resuscitation now recommends this practice. ${ }^{3}$

received

February 1, 2017

accepted after revision

May 3, 2017

published online

June 7, 2017
The purpose of this review is to present the evidence in support of delayed clamping of the umbilical cord. First, we will discuss the effects of delayed cord clamping or cord milking on the transitional physiology of neonates. Then, we will review the effects of delayed cord clamping on neonatal outcomes.

\section{Delayed Cord Clamping and Transitional Physiology}

One needs to understand the physiology of cardiovascular and respiratory transition as a foundation for understanding the role of placental transfusion that results from delayed cord clamping. There are several aspects of transitional physiology to be considered including effects of lung expansion, changes in the distribution of cardiac output, and blood volume shifts that could benefit from placental transfusion and the possible benefits of the transfusion of stem cells into the newborn.

The fetal circulation allows for only 7 to $8 \%$ of cardiac output into the lungs. ${ }^{4,5}$ Over $90 \%$ of cardiac output supplies the systemic circulation, of which $35 \%$ comes from left ventricular output and the remainder through the fetal shunt supplied by the patent ductus arteriosus (PDA) ${ }^{6}$ as shown

Copyright $\odot 2017$ by Thieme Medical Publishers, Inc., 333 Seventh Avenue, New York, NY 10001, USA.

DOI https://doi.org/ 10.1055/s-0037-1603733. Tel: +1(212) 584-4662. 
in -Fig. 1A. Since the placenta is the organ of gas exchange, the fetal lungs do not require much blood flow in utero. Preload to the left ventricle (LV) of the fetus is provided by the flow of blood from the umbilical vein through the foramen ovale into the left atrium $^{7}$ (- Fig. 1A).

At birth, there is a significant increase in the blood flow to the lungs as they assume the responsibility for gas exchange with a rapid decrease in pulmonary vascular resistance related to many factors such as aeration, oxygenation, prostaglandin E, and stimulation of vagal fibers in response to interstitial fluid. ${ }^{6,8-10}$ Right ventricular output to the pulmonary circulation increases from 8 to $45 \%$ immediately after birth, which further increases to $100 \%$ of cardiac output in the adult circulation after fetal shunts have closed. This increased blood volume comes from the systemic circulation if there is a disruption of flow from the placenta when the umbilical cord is clamped immediately at birth. The potential for development of systemic hypoperfusion can lead to hypotension as shown in - Fig. $\mathbf{1 B}$.

\section{Animal Studies}

Recent work in the laboratory has documented the effect of these blood flow changes at birth. Immediately after birth, the lungs become aerated resulting in a large increase in pulmonary blood flow. ${ }^{7,11,12}$ Both ventilation and oxygenation lead to a decrease in pulmonary vascular resistance and increased pulmonary blood flow after birth. ${ }^{12}$ The left-toright flow through the PDA that occurs within minutes after
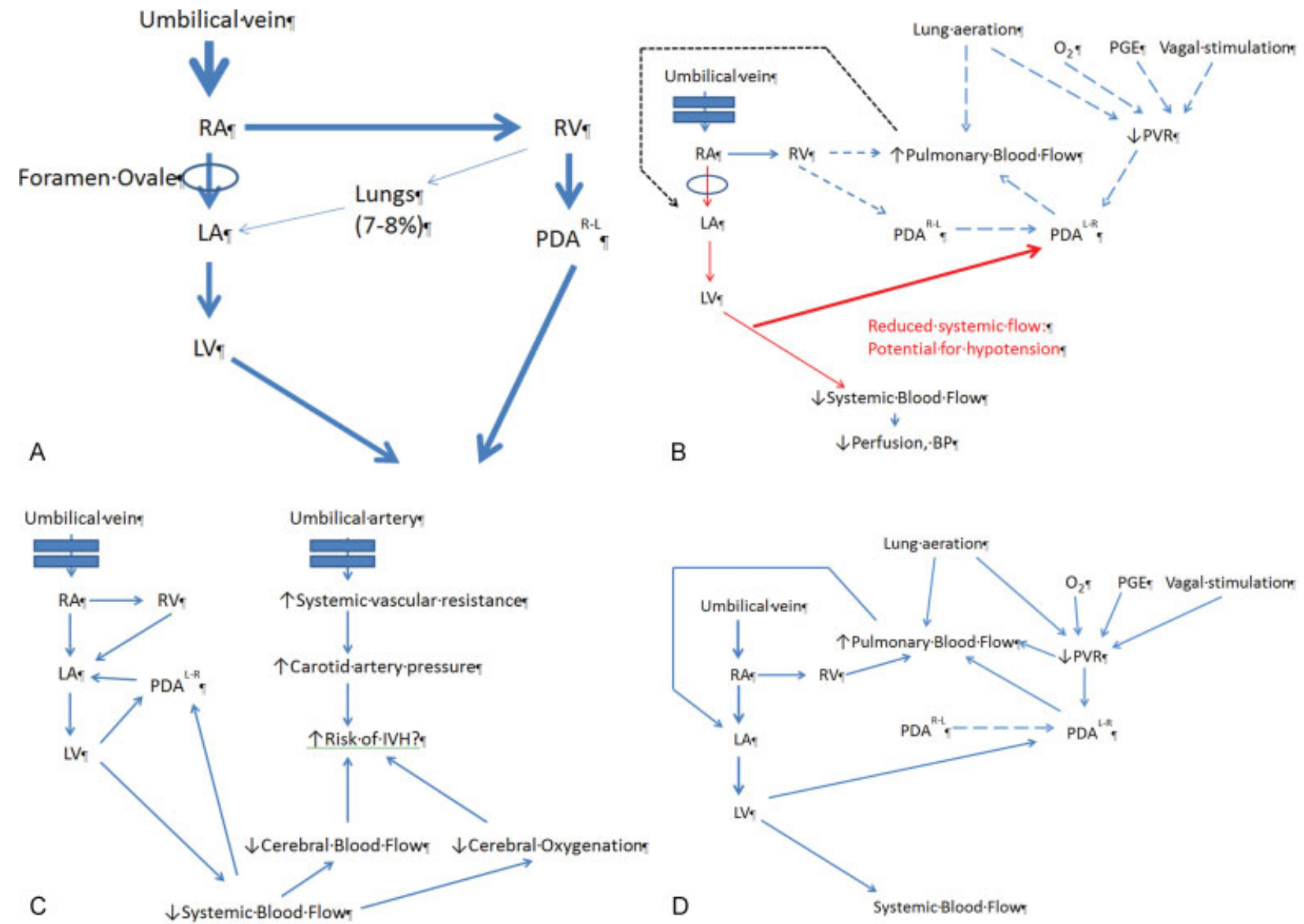

Fig. 1 (A) Fetal circulation is shown in the model depicting the fetal shunts that supply systemic circulation. As only 7 to $8 \%$ of cardiac output goes to the pulmonary circulation, most of the RV output passes through the PDA to supply systemic blood flow. (B) Disruption in the umbilical venous flow that occurs with immediate cord clamping shows the downstream effects on systemic blood flow, whereby preload to the LV is reduced, and systemic circulation and perfusion are reduced (thin red arrows). Lung inflation after cord clamping delays the increase in pulmonary blood flow that provides LA return and left LV preload (dashed arrows). The thick red arrow indicates the "steal" of blood flow from the systemic circulation associated with the reversal of the shunt through the PDA from right-to-left to left-to-right, which supplies blood volume when the pulmonary circulation is established. (C) Disruption of umbilical arterial flow as well as umbilical venous flow is shown that occurs with cord clamping. Removing the low-resistance, high-flow placental circulation from the systemic circulation by clamping the umbilical artery increases the systemic vascular resistance, which elevates the carotid artery pressure. The elevated carotid artery pressure, along with reduction in systemic blood flow that results when the umbilical vein is immediately clamped, can increase the risk of IVH in preterm infants. (D) The model shows the circulatory support of systemic circulation when the umbilical cord is not clamped immediately. When ventilation of the newborn's lungs occurs before umbilical cord clamping, the increased blood flow from the umbilical venous return counterbalances the loss of systemic flow that occurs through the PDA. This provides the preload needed for the left ventricle (LV) that will subsequently be provided by the pulmonary venous return associated with the decrease in pulmonary vascular resistance that occurs in the first 3 minutes after birth. LA, left atrial; LV, left ventricle; PDA, patent ductus arteriosus; RV, right ventricular. 
birth contributes to approximately $50 \%$ of the increase in pulmonary blood flow. ${ }^{13}$ Thus, much of the increased pulmonary blood flow that occurs in the first 3 minutes after birth occurs at the expense of the systemic circulation from the LV through the PDA (-Fig. 1B). Clamping the umbilical cord at birth eliminates umbilical venous return and results in a sudden 30 to $50 \%$ decrease in both left and right ventricular preload. $^{13,14}$ Simultaneously, cord clamping increases systemic vascular resistance and afterload by removing the low-resistance, high-flow placental circulation. The decrease in ventricular preload along with the increase in left ventricular afterload after immediate umbilical cord clamping before lung inflation results in a decrease in both left and right ventricular output. This can have deleterious effects on systemic and vital organ perfusion. A recent study in preterm lambs showed that clamping the umbilical cord prior to providing ventilation caused a rapid decrease in both systemic saturation of peripheral oxygen $\left(\mathrm{SpO}_{2}\right)$ and cerebral regional oxygenation. ${ }^{15}$ At the same time, there was a sharp rise in carotid arterial pressure and flow when the umbilical cord was clamped prior to providing ventilation, ${ }^{15}$ which is depicted in - Fig. 1C.

The increase in pulmonary blood flow with lung aeration provides left ventricular preload, replacing the blood flow from the placenta. The increase in pulmonary blood flow is more likely due to the response to increased oxygenation and not due to lung expansion, as studies have shown a marked mismatch in ventilation and perfusion with nonaerated regions, demonstrating a similar increase in blood flow to aerated regions of the lung. ${ }^{6,9}$ Polglase and colleagues recently showed that ventilation prior to clamping of the umbilical cord of preterm lambs enhanced systemic and cerebral oxygenation compared with immediate umbilical cord clamping followed by ventilation. ${ }^{15}$ Thus, ventilation prior to cord clamping (delayed clamping) can result in more stable hemodynamics by providing the left atrial and left ventricular preload that will replace the blood flow provided by the umbilical vein when the umbilical cord is clamped, thereby reducing the chance of systemic hypotension (-Fig. 1D).

\section{Clinical Studies}

Studies in human infants have shown that there is a significant increase in risk of death or need for critical care when cord clamping occurred before onset of spontaneous respiration. ${ }^{16}$ It is more physiological to delay cord clamping until the lungs have been inflated. Delayed cord clamping was shown to be associated with increased antioxidant defenses (increased superoxide dismutase and catalase activities) in cord blood, thereby increasing antioxidant capacity and reducing inflammation-mediated pathways that are activated by delivery. ${ }^{17}$ Delayed cord clamping in preterm human infants was shown to be associated with higher superior vena cava blood flow and improved right ventricular stroke volume and output. ${ }^{18}$ In a quality improvement(QI) audit study, delayed cord clamping in preterm infants was well tolerated with no adverse effects on the need for resuscitation at birth, Apgar scores, admission temperatures, and base deficits compared with preterm infants born after immediate cord clamping (prior to implementation of the practice of delayed cord clamping). ${ }^{19}$ These data from laboratory and human studies support the concept that when the newborn is resuscitated using ventilation prior to umbilical cord clamping, the systemic perfusion and blood pressure are maintained and vital organ perfusion is optimal.

\section{Effects of Delayed Cord Clamping on Neonatal Outcomes}

\section{Term Infants}

Studies of delayed umbilical cord clamping in term infants defined late clamping as waiting until umbilical arterial pulsations cease. In a study of term, uncomplicated pregnancies, late cord clamping resulted in higher systolic blood pressures in the first 24 hours after birth, and these infants had a later-onset of spontaneous respiration compared with term infants, in whom the umbilical cord was clamped immediately after delivery. ${ }^{20}$ The same group found that infants who had delayed cord clamping also had higher blood volume, urine output, and effective renal blood flow in the first 12 hours after birth. ${ }^{21}$

A more recent study, using a randomized controlled study design, compared term, uncomplicated infants with early cord clamping (within 10 seconds of birth) to delayed cord clamping (defined as 3 minutes after birth or after cessation of umbilical arterial pulsation) with a primary outcome of infants' hemoglobin and iron status at 4 months of age. The delayed cord clamping group had higher serum ferritin and less iron deficiency but no difference in respiratory symptoms or need for phototherapy in the newborn period. ${ }^{22} \mathrm{~A}$ follow-up study by this group showed that the infants born after delayed cord clamping had better fine motor function and social development at 4 years of age compared with infants born after immediate cord clamping. ${ }^{23}$

A meta-analysis of studies comparing late and early umbilical cord clamping showed that delayed cord clamping increased the relative risk (RR) of polycythemia (defined as hematocrit $>65 \%$ ) by 3.8 -fold at 24 to 48 hours of age. ${ }^{24}$ This analysis, however, showed that the RR of anemia at 2 to 3 months of age was reduced by $47 \%$ (RR ratio, 0.53 ) by delayed cord clamping. ${ }^{24}$ The RR for clinical jaundice at 24 to 48 hours of age was increased by $35 \%$ in neonates who had late cord clamping compared with early cord clamping, but there was no increase in the need for phototherapy. ${ }^{24} \mathrm{~A}$ more recent metaanalysis, however, showed that the RR for jaundice requiring phototherapy was increased in term infants born after delayed cord clamping. ${ }^{25}$

Delayed cord clamping does not increase the risk of maternal postpartum hemorrhage, maternal transfusion requirements, need for manual extraction of the placenta, and length of the third stage of labor, and does not decrease maternal hemoglobin. ${ }^{25,26}$ In healthy full-term infants, delayed cord clamping ( 2 minutes) did not change acidbase status, maternal hematocrit, and hemoglobin, and did not increase the duration of the third stage of labor compared with term infants who had early cord clamping. 27

Therefore, delayed cord clamping in term, uncomplicated pregnancies improves systemic perfusion as evidenced by 
higher blood pressure, blood volume, and urine output in the first 24 hour after birth, and reduces the risk of iron deficiency anemia in infancy ( - Table $\mathbf{1}$ ). Iron deficiency anemia is a significant concern, especially in low-resource settings. Preventing or reducing the risk of iron deficiency anemia may have long-term benefits in terms of neurodevelopment. Delayed cord clamping in the term infant slightly increases the need for phototherapy of neonatal jaundice (risk difference of $<2 \%$, and does not increase the risk of maternal postpartum hemorrhage or the duration of the third stage of labor (-Table 1). While delayed cord clamping does increase the risk of neonatal polycythemia without an increase in the need for treatment, there are no data on the long-term outcomes related to delayed cord clamping-associated polycythemia in term infants.

\section{Preterm Infants}

A randomized controlled trial of late preterm infants (34-36 weeks' gestation at birth) found that delayed cord clamping, defined as clamping of the umbilical cord 3 minutes after birth, was associated with higher neonatal hemoglobin at birth and at 10 weeks of age compared with infants with early cord clamping (within 30 seconds after birth). ${ }^{28}$ There were no differences in the blood glucose levels, polycythemia or need for phototherapy between the groups. ${ }^{28}$ A recently published randomized controlled trial showed that late preterm and term infants born after delayed cord clamping ( $>3$ minutes) had higher hemoglobin levels and a lower prevalence of iron deficiency anemia at 8 and 12 months of age than infants born after early cord clamping ( $<60$ seconds). ${ }^{29}$ The number needed to treat was 6 to reduce iron deficiency anemia at 8 months of age, and the number needed to treat was 12 to reduce the risk of anemia at 8 and 12 months of age. ${ }^{29}$

Preterm infants (30-36 weeks' gestation at birth) randomized to delayed cord clamping (at 1 minute of age) had larger circulating red blood cell (RBC) volume, no difference in hematocrit at birth, but higher hematocrit at 7,14,21, and
28 days of age compared with preterm infants randomized to immediate cord clamping within 5 seconds of birth. ${ }^{30,31}$ There was no increase in polycythemia or jaundice in the delayed cord clamping groups. ${ }^{31}$ Another randomized controlled trial found that preterm infants assigned to delayed cord clamping (30-90 seconds after birth) had significantly higher blood volumes regardless of mode of delivery (cesarean section or vaginal delivery) compared with infants who had immediate cord clamping. ${ }^{32}$ Kinmond and colleagues reported that preterm infants randomized to "regulated" cord clamping (delay for 30 seconds with the infant held below the introitus) had higher hematocrits without polycythemia, a lower requirement for blood transfusion, and spent less time on supplemental oxygen compared with preterm infants assigned to "random" cord clamping (within 10 seconds of birth and not held below the introitus). ${ }^{33}$ There was no difference in Apgar scores or in admission temperatures between the groups. ${ }^{33}$

Rabe and colleagues found that preterm infants born at fewer than 33 completed weeks of gestation and who were randomized to delayed cord clamping for 45 seconds had significantly higher hemoglobin in the first 28 days of age with a $50 \%$ reduction in the need for packed RBC transfusion compared with preterm infants who had delayed cord clamping for 20 seconds. ${ }^{34}$ There was no difference between the groups in phlebotomy blood loss. ${ }^{34}$

A small pilot study, using a randomized controlled design, in extremely low-birth weight preterm infants (24-32 completed weeks of gestation) showed that delayed cord clamping (30-45 seconds after birth) was safe with no increase in risk of hypothermia, hyperbilirubinemia, and no delay in intubation compared with similar preterm infants who underwent immediate cord clamping. ${ }^{35}$ The delayed cord clamping group had a higher initial mean blood pressure, higher initial blood glucose, and less hypoglycemia. ${ }^{35}$ A larger trial of preterm infants born at fewer than 32 completed weeks of gestation showed that infants randomized to delayed cord clamping

Table 1 Benefits of delayed cord clamping in term and preterm newborn infants

\begin{tabular}{|c|c|}
\hline Term newborns & Preterm newborns \\
\hline Higher blood pressure in first 24 hours $^{20}$ & Higher hemoglobin at birth, 10 weeks of age ${ }^{29,41}$ \\
\hline Higher blood volume in first 12 hours $^{21}$ & Larger circulating RBC volume at birth ${ }^{31}$ \\
\hline Higher urine output in first 12 hours $^{21}$ & Higher hematocrit at 7 to 28 days of age $\mathrm{e}^{31,32}$ \\
\hline Higher hemoglobin at 4 months of age ${ }^{22}$ & Higher blood volume at birth ${ }^{33}$ \\
\hline Lower incidence of iron deficiency ${ }^{22}$ & Higher hematocrit without polycythemia at birth ${ }^{34}$ \\
\hline Slight increase in need for phototherapy ${ }^{25}$ & Less need for blood transfusion ${ }^{34,35}$ \\
\hline No increase in the need to treat polycythemia ${ }^{24,25}$ & Less time on supplemental oxygen ${ }^{34}$ \\
\hline \multirow[t]{5}{*}{ No increase in maternal morbidity ${ }^{25,27}$} & Higher initial mean blood pressure ${ }^{36}$ \\
\hline & Lower incidence of hypoglycemia $^{36}$ \\
\hline & Lower incidence of late-onset sepsis ${ }^{37}$ \\
\hline & Lower incidence of $\mathrm{IVH}^{37,38}$ \\
\hline & Improved PDI on BSID in male preterm infants ${ }^{39}$ \\
\hline
\end{tabular}

Abbreviations: BSID, Bayley scales of infant development; IVH, intraventricular hemorrhage; PDI, psychomotor developmental index; RBC, red blood cells. 
(30-45 seconds after birth with the infant held 10 to15 inches below the introitus or incision) had lower incidence of intraventricular hemorrhage (IVH) and late-onset sepsis compared with infants assigned to the immediate cord clamping group. ${ }^{36}$ There were no differences in the incidences of necrotizing enterocolitis, bronchopulmonary dysplasia (BPD), or retinopathy of prematurity between the groups. ${ }^{36} \mathrm{~A} \mathrm{QI}$ audit of outcomes of preterm infants ( $<35$ weeks' gestation) found that implementation of the practice of delayed cord clamping (30-60 seconds) was associated with a decrease in the incidence of IVH, an increase in the initial hematocrit of the newborn, and improved admission temperature. ${ }^{37}$

Long-term neurodevelopmental outcome at 7 months of age was improved in infants assigned to delayed cord clamping. ${ }^{38}$ Infants whose umbilical cords were clamped 30 to 45 seconds after birth had improved psychomotor developmental index (PDI) on Bayley scales of infant development (BSID) testing, an effect seen in male preterm infants but not female preterm infants. ${ }^{38}$ A recent study by this group found that delayed cord clamping (30-45 seconds) followed by one cord milking reduced the rates of poor motor performance at 18 to 22 months of age in preterm infants compared with infants whose umbilical cords were immediately clamped after birth. $^{39}$

Oh and colleagues reported a randomized controlled trial of preterm infants born at fewer than 28 completed weeks' gestation and found that infants assigned to delayed cord clamping (30-45 seconds) had higher venous hematocrits with no difference in mean arterial blood pressures and no difference in delivery room management and intervention compared with preterm infants who had the umbilical cord clamped immediately after birth. ${ }^{40}$

In a QI report of a change in obstetrical practice, delayed cord clamping (45 seconds after birth with the infant held 10 to $20 \mathrm{~cm}$ below the placenta) was associated with less need for resuscitation in the delivery room and higher hematocrit in very low-birth weight infants (birth weight 401-1,500 g) compared with historical controls who had immediate cord clamping. ${ }^{41}$ Delayed cord clamping was associated with higher blood pressure in very low-birth weight infants with no difference in neonatal hyperbilirubinemia or in the need for phototherapy compared with the historical controls. ${ }^{41}$

In a prospective cohort study nested within a randomized controlled trial of extremely preterm infants, delayed cord clamping was shown to result in higher superior vena cava (SVC) flow from 6 hours to 4 days of age and higher RV stroke volume and output compared with infants born followed by immediate cord clamping. ${ }^{18}$ Improved flow in the SVC may maintain cerebral autoregulation of blood flow, which may be one of the mechanisms by which delayed cord clamping reduces the risk of IVH in preterm infants.

Therefore, there are numerous benefits of delayed cord umbilical clamping in the preterm infant as shown in - Table 1.

\section{Umbilical Cord Milking}

Because umbilical cord milking is faster than delayed cord clamping, it alleviates concerns regarding a delay in neonatal resuscitation. In a randomized controlled trial of extremely premature infants (24-28 weeks' gestation), assignment to cord milking was associated with higher 1 minute Apgar scores, decreased need for postnatal transfusion, higher hemoglobin levels, higher blood pressure, and shorter duration of mechanical ventilation and days in supplemental oxygen compared with the group who had immediate umbilical cord clamping. ${ }^{42}$ There was no polycythemia in the infants whose umbilical cords were milked and there was no difference in the bilirubin levels between the two groups. ${ }^{42} \mathrm{~A}$ secondary analysis of this study revealed that the infants randomized to cord milking had a reduced need for volume expansion and inotropic support, and had a higher urine output in the first 72 hours after birth compared with the neonates whose umbilical cords were immediately clamped at birth. ${ }^{43}$ A retrospective cohort study of infants born at fewer than 29 completed weeks of gestation showed that umbilical cord milking was associated with higher hemoglobin levels, mean arterial blood pressure, lower need for volume expansion, higher urine output in the first 24 hours after birth, higher SVC blood flow, better left ventricular cardiac output, and improved cerebral oxygenation compared with immediate cord clamping. ${ }^{44}$

Rabe and colleagues conducted a randomized controlled trial of premature infants born at 24 to 33 weeks' gestation comparing umbilical cord milking and delayed cord clamping (defined as 30 seconds after birth). ${ }^{45}$ They found no differences in Apgar scores, admission temperatures, mean arterial blood pressures, hemoglobin levels, need for transfusion, and mortality or morbidity (BPD, necrotizing enterocolitis [NEC], IVH, retinopathy of prematurity [ROP], or sepsis) between the groups. ${ }^{45}$

A recent two-center randomized controlled trial of infants born at fewer than 32 weeks of gestation compared umbilical cord milking with delayed cord clamping (defined as clamping of the cord at 45 to 60 seconds after birth) and found that the infants who were delivered by cesarean birth and assigned to umbilical cord milking had higher SVC blood flow and right ventricular output in the first 12 hours after birth. ${ }^{46}$ Also, these infants had higher hemoglobin levels at birth, delivery room temperature, and blood pressure in the first 15 hours after birth, and higher urine output in the first 24 hours after birth. ${ }^{46}$

A recent systematic review and meta-analysis of the efficacy and safety of umbilical cord milking compared with either immediate or delayed cord clamping found that umbilical cord milking was associated with some benefits without adverse effects. ${ }^{47}$ Cord milking significantly decreased the RR of an oxygen requirement at 36 weeks' postmenstrual age as well as a decrease in the risk of IVH. ${ }^{47}$ However, there was heterogeneity in that the included studies in this analysis used either immediate or delayed cord clamping as the comparison group. Further studies will be needed to compare delayed cord clamping with cord milking since most of the studies included in this metaanalysis used immediate cord clamping as the control group. There is a theoretical risk of increased IVH in the preterm infant if cord milking is performed before the pulmonary 
vascular resistance is lowered by lung inflation. In this situation, cord milking will increase preload to the left ventricle without the ability to increase flow to the lungs through the PDA so that carotid flow and pressure would increase pathologically, resulting in an increased risk of IVH.

\section{Systematic Reviews and Meta-analysis of Delayed Cord Clamping}

In term newborns, delayed cord clamping improves hemoglobin and hematocrit levels in the first 5 days after birth and in the first 2 to 3 months of age with improved iron status and less anemia at 2 to 3 months of age compared with immediate cord clamping. ${ }^{24}$ There is an increase in clinical jaundice in term infants with a small increase in the need for phototherapy when there is a delay in cord clamping $(<2 \%$ risk difference). ${ }^{25}$ Term infants have a higher risk of polycythemia (hematocrit $>65 \%$ ) associated with delayed cord clamping, but there is no increase in the need for partial reduction exchange transfusion. ${ }^{24}$ Thus, while there are some concerns about the risks for jaundice and polycythemia with delayed cord clamping in term infants, there does not appear to be an increase in the treatment for polycythemia. Delayed cord clamping after term birth is recommended as long as there are capabilities to provide phototherapy, if needed.

Delayed cord clamping in the preterm neonate is not harmful as there is no effect on Apgar scores, admission temperatures, need for resuscitation, incidence of respiratory distress syndrome and polycythemia, and severity of hyperbilirubinemia. ${ }^{48}$ Placental transfusion from delay in clamping the umbilical cord improves outcomes including higher blood pressures and blood volumes, less need for inotropes, higher hematocrit in the first 24 hours after birth, less need for packed RBC transfusions, and most importantly, reduced risk of IVH and NEC. ${ }^{48}$

\section{Conclusion}

There is ample evidence in the literature over the last 50 years that delayed umbilical cord clamping in both term and preterm infants is beneficial for short- and long-term outcomes. Recent studies in the laboratory indicate that providing ventilation in the initial steps of neonatal resuscitation prior to clamping of the umbilical cord has a physiologic basis and will result in better outcomes for newborns, especially those born prematurely. The challenge now is to design equipment and strategies that can allow initial resuscitation very close to the mother while the umbilical cord is still intact and attached to the placenta. Pilot studies indicate that this is feasible. ${ }^{49}$ While umbilical cord milking may be an alternative, more studies are needed to determine if this approach will yield similar benefits to delayed cord clamping.

\footnotetext{
Acknowledgment

The author thanks Dr. Charles Palmer for his review of and thoughtful suggestions for the article.
}

\section{References}

1 Beck AC. How can the obstetrician aid in reducing the mortality of prematurely born infants? Am J Ob Gyn 1941;42(02):355-364

2 Committee on Obstetric Practice. Committee opinion No. 684: delayed umbilical cord clamping after birth. Obstet Gynecol 2017; 129(01):e5-e10

3 Textbook of Neonatal Resuscitation. 7th ed. In: Weiner GM, Zaichkin J eds. Elk Grove Village, IL: American Academy of Pediatrics and American Heart Association; 2016

4 Swanson JR, Sinkin RA. Transition from fetus to newborn. Pediatrv Clin North Am 2015;62(02):329-343

5 Dawes GS. Changes in the circulation at birth. Br Med Bull 1961; 17(02):148-153

6 Lang JA, Pearson JT, te Pas AB, et al. Ventilation/perfusion mismatch during lung aeration at birth. J Appl Physiol (1985) 2014; 117(05):535-543

7 Hooper SB, Polglase GR, Roehr CC. Cardiopulmonary changes with aeration of the newborn lung. Paediatr Respir Rev 2015;16(03): $147-150$

8 Iwamoto HS, Teitel D, Rudolph AM. Effects of birth-related events on blood flow distribution. Pediatr Res 1987;22(06): 634-640

9 Lang JA, Pearson JT, Binder-Heschl C, et al. Increase in pulmonary blood flow at birth: role of oxygen and lung aeration. J Physiol 2016;594(05):1389-1398

10 Rudolph AM. Fetal and neonatal pulmonary circulation. Annu Rev Physiol 1979;41:383-395

11 Cassin S, Dawes GS, Mott JC, Ross BB, Strang LB. The vascular resistance of the foetal and newly ventilated lung of the lamb. J Physiol 1964;171:61-79

12 Teitel DF, Iwamoto HS, Rudolph AM. Changes in the pulmonary circulation during birth-related events. Pediatr Res 1990; 27(4, Pt 1):372-378

13 Crossley KJ, Allison BJ, Polglase GR, Morley CJ, Davis PG, Hooper SB. Dynamic changes in the direction of blood flow through the ductus arteriosus at birth. J Physiol 2009;587(Pt 19):46954704

14 Bhatt S, Alison BJ, Wallace EM, et al. Delaying cord clamping until ventilation onset improves cardiovascular function at birth in preterm lambs. J Physiol 2013;591(08):2113-2126

15 Polglase GR, Dawson JA, Kluckow M, et al. Ventilation onset prior to umbilical cord clamping (physiological-based cord clamping) improves systemic and cerebral oxygenation in preterm lambs. PLoS One 2015;10(02):e0117504. Doi: 10.1371/journal.pone.0117504

16 Ersdal HL, Linde J, Mduma E, Auestad B, Perlman J. Neonatal outcome following cord clamping after onset of spontaneous respiration. Pediatrics 2014;134(02):265-272

17 Díaz-Castro J, Florido J, Kajarabille N, et al. The timing of cord clamping and oxidative stress in term newborns. Pediatrics 2014; 134(02):257-264

18 Sommers R, Stonestreet BS, Oh W, et al. Hemodynamic effects of delayed cord clamping in premature infants. Pediatrics 2012; 129(03):e667-e672

19 Nevill E, Meyer MP. Effect of delayed cord clamping (DCC) on breathing and transition at birth in very preterm infants. Early Hum Dev 2015;91(07):407-411

$20 \mathrm{Oh} \mathrm{W}$, Lind J, Gessner IH. The circulatory and respiratory adaptation to early and late cord clamping in newborn infants. Acta Paediatr Scand 1966;55(01):17-25

21 Oh W, Oh MA, Lind J. Renal function and blood volume in newborn infant related to placental transfusion. Acta Paediatr Scand 1966; 56:197-210

22 Andersson O, Hellström-Westas L, Andersson D, Domellöf M. Effect of delayed versus early umbilical cord clamping on neonatal outcomes and iron status at 4 months: a randomised controlled trial. BMJ 2011;343:d7157-d7163

23 Andersson O, Lindquist B, Lindgren M, Stjernqvist K, Domellöf M, Hellström-Westas L. Effect of delayed cord clamping on 
neurodevelopment at 4 years of age: a randomized clinical trial. JAMA Pediatr 2015;169(07):631-638

24 Hutton EK, Hassan ES. Late vs early clamping of the umbilical cord in full-term neonates: systematic review and meta-analysis of controlled trials. JAMA 2007;297(11):1241-1252

25 McDonald SJ, Middleton P, Dowswell T, Morris PS. Effect of timing of umbilical cord clamping of term infants on maternal and neonatal outcomes. Cochrane Database Syst Rev 2013;(07): CD004074. Doi: 10.1002/14651858.CD004074.pub3

26 McDonald SJ, Middleton P. Effect of timing of umbilical cord clamping of term infants on maternal and neonatal outcomes. Cochrane Database Syst Rev 2008;2(02):CD004074. Doi: 10.1002/ 14651858.CD004074.pub2

27 De Paco C, Herrera J, Garcia C, et al. Effects of delayed cord clamping on the third stage of labour, maternal haematological parameters and acid-base status in fetuses at term. Eur J Obstet Gynecol Reprod Biol 2016;207:153-156

28 Ultee CA, van der Deure J, Swart J, Lasham C, van Baar AL. Delayed cord clamping in preterm infants delivered at 3436 weeks' gestation: a randomised controlled trial. Arch Dis Child Fetal Neonatal Ed 2008;93(01):F20-F23

29 Kc A, Rana N, Målqvist M, Jarawka Ranneberg L, Subedi K, Andersson O. Effects of delayed umbilical cord clamping vs early clamping on anemia in infants at 8 and 12 months: a randomized clinical trial. JAMA Pediatr 2017;171(03):264-270

30 Strauss RG, Mock DM, Johnson K, et al. Circulating RBC volume, measured with biotinylated RBCs, is superior to the Hct to document the hematologic effects of delayed versus immediate umbilical cord clamping in preterm neonates. Transfusion 2003; 43(08):1168-1172

31 Strauss RG, Mock DM, Johnson KJ, et al. A randomized clinical trial comparing immediate versus delayed clamping of the umbilical cord in preterm infants: short-term clinical and laboratory endpoints. Transfusion 2008;48(04):658-665

32 Aladangady N, McHugh S, Aitchison TC, Wardrop CA, Holland BM. Infants' blood volume in a controlled trial of placental transfusion at preterm delivery. Pediatrics 2006;117(01):93-98

33 Kinmond S, Aitchison TC, Holland BM, Jones JG, Turner TL, Wardrop CA. Umbilical cord clamping and preterm infants: a randomised trial. BMJ 1993;306(6871):172-175

34 Rabe H, Wacker A, Hülskamp G, et al. A randomised controlled trial of delayed cord clamping in very low birth weight preterm infants. Eur J Pediatr 2000;159(10):775-777

35 Mercer JS, McGrath MM, Hensman A, Silver H, Oh W. Immediate and delayed cord clamping in infants born between 24 and 32 weeks: a pilot randomized controlled trial. J Perinatol 2003; 23(06):466-472

36 Mercer JS, Vohr BR, McGrath MM, Padbury JF, Wallach M, Oh W. Delayed cord clamping in very preterm infants reduces the incidence of intraventricular hemorrhage and late-onset sepsis: a randomized, controlled trial. Pediatrics 2006;117(04):1235-1242
37 Jelin AC, Zlatnik MG, Kuppermann M, Gregorich SE, Nakagawa S, Clyman R. Clamp late and maintain perfusion (CLAMP) policy: delayed cord clamping in preterm infants. J Matern Fetal Neonatal Med 2016;29(11):1705-1709

38 Mercer JS, Vohr BR, Erickson-Owens DA, Padbury JF, Oh W. Sevenmonth developmental outcomes of very low birth weight infants enrolled in a randomized controlled trial of delayed versus immediate cord clamping. J Perinatol 2010;30(01):11-16

39 Mercer JS, Erickson-Owens DA, Vohr BR, et al. Effects of placental transfusion on neonatal and 18 month outcomes in preterm infants: a randomized controlled trial. J Pediatr 2016;168: 50-55.e1

40 Oh W, Fanaroff AA, Carlo WA, Donovan EF, McDonald SA, Poole WK; Eunice Kennedy Shriver National Institute of Child Health and Human Development Neonatal Research Network. Effects of delayed cord clamping in very-low-birth-weight infants. J Perinatol 2011;31(Suppl 1):S68-S71

41 Kaempf JW, Tomlinson MW, Kaempf AJ, et al. Delayed umbilical cord clamping in premature neonates. Obstet Gynecol 2012; 120(2, Pt 1):325-330

42 Hosono S, Mugishima H, Fujita $\mathrm{H}$, et al. Umbilical cord milking reduces the need for red cell transfusions and improves neonatal adaptation in infants born at less than 29 weeks' gestation: a randomised controlled trial. Arch Dis Child Fetal Neonatal Ed 2008;93(01):F14-F19

43 Hosono S, Mugishima H, Fujita $\mathrm{H}$, et al. Blood pressure and urine output during the first $120 \mathrm{~h}$ of life in infants born at less than 29 weeks' gestation related to umbilical cord milking. Arch Dis Child Fetal Neonatal Ed 2009;94(05):F328-F331

44 Takami T, Suganami Y, Sunohara D, et al. Umbilical cord milking stabilizes cerebral oxygenation and perfusion in infants born before 29 weeks of gestation. J Pediatr 2012;161(04):742-747

45 Rabe H, Jewison A, Alvarez RF, et al; Brighton Perinatal Study Group. Milking compared with delayed cord clamping to increase placental transfusion in preterm neonates: a randomized controlled trial. Obstet Gynecol 2011;117(2, Pt 1):205-211

46 Katheria AC, Truong G, Cousins L, Oshiro B, Finer NN. Umbilical cord milking versus delayed cord clamping in preterm infants. Pediatrics 2015;136(01):61-69

47 Al-Wassia H, Shah PS. Efficacy and safety of umbilical cord milking at birth: a systematic review and meta-analysis. JAMA Pediatr 2015;169(01):18-25

48 Rabe H, Diaz-Rossello JL, Duley L, Dowswell T. Effect of timing of umbilical cord clamping and other strategies to influence placental transfusion at preterm birth on maternal and infant outcomes. Cochrane Database Syst Rev 2012;(08):CD003248. Doi: 10.1002/14651858.CD003248.pub3

49 Winter J, Kattwinkel J, Chisholm C, Blackman A, Wilson S, Fairchild $\mathrm{K}$. Ventilation of preterm infants during delayed cord clamping (VentFirst): a pilot study of feasibility and safety. Am J Perinatol 2017;34(02):111-116 